\title{
Evidence for two types of brown adipose tissue in humans
}

Martin E. Lidell, Matthias J. Betz, Olof Dahlqvist Leinhard, Mikael Heglind, Louise Elander, Marc Slawik, Thomas Mussack, Daniel Nilsson, Thobias Romu, Pirjo Nuutila, Kirsi A.

Virtanen, Felix Beuschlein, Anders Persson, Magnus Borga and Sven Enerbäck

\section{Linköping University Post Print}

\section{Tweet}

N.B.: When citing this work, cite the original article.

Original Publication:

Martin E. Lidell, Matthias J. Betz, Olof Dahlqvist Leinhard, Mikael Heglind, Louise Elander, Marc Slawik, Thomas Mussack, Daniel Nilsson, Thobias Romu, Pirjo Nuutila, Kirsi A. Virtanen, Felix Beuschlein, Anders Persson, Magnus Borga and Sven Enerbäck, Evidence for two types of brown adipose tissue in humans, 2013, Nature Medicine, (19), 5, 631-634.

http://dx.doi.org/10.1038/nm.3017

Copyright: Nature Publishing Group http://www.nature.com/

Postprint available at: Linköping University Electronic Press http://urn.kb.se/resolve?urn=urn:nbn:se:liu:diva-91307 


\section{Evidence for Two Types of Brown Adipose Tissue in Humans}

Martin E. Lidell ${ }^{1 *}$, Matthias J. Betz ${ }^{1,2^{*}}$, Olof Dahlqvist Leinhard ${ }^{3,4}$, Mikael Heglind ${ }^{1}$, Louise

Elander $^{3,5}$, Marc Slawik ${ }^{2}$, Thomas Mussack ${ }^{6}$, Daniel Nilsson ${ }^{1}$, Thobias Romu ${ }^{3,7}$, Pirjo

Nuutila $^{8}$, Kirsi A. Virtanen ${ }^{8}$, Felix Beuschlein ${ }^{2}$, Anders Persson ${ }^{3,5}$, Magnus Borga ${ }^{3,7}$ and

Sven Enerbäck ${ }^{1}$

${ }^{1}$ Department of Medical and Clinical Genetics, Institute of Biomedicine, The Sahlgrenska Academy, University of Gothenburg, Gothenburg, Sweden; ${ }^{2}$ Endocrine Research Unit, Medizinische Klinik und Poliklinik IV, Klinikum der LMU, Munich, Germany; ${ }^{3}$ Center for Medical Image Science and Visualization (CMIV), Linköping University, Linköping, Sweden; ${ }^{4}$ Department of Medical and Health Sciences (IMH), Linköping University, Linköping, Sweden; ${ }^{5}$ University Hospital, County Council of Östergötland, Linköping, Sweden; ${ }^{6}$ Department of Surgery Innenstadt, Klinikum der LMU, Munich, Germany;

${ }^{7}$ Department of Biomedical Engineering (IMT), Linköping University, Linköping, Sweden; ${ }^{8}$ Turku PET Center, University of Turku, Turku, Finland

* Drs. Lidell and Betz contributed equally to this article.

Corresponding Author: $\quad$ Sven Enerbäck, MD, PhD

E-mail: sven.enerback@medgen.gu.se

Phone: $+46-31-7863334$

Fax: $+46-31-416108$ 
The previously observed supraclavicular depot of brown adipose tissue (BAT) in adult humans was commonly believed to be the equivalent of the interscapular thermogenic organ of small mammals. This view was very recently disputed by $\mathrm{Wu}$ et al., based on the demonstration that this depot is made up by beige or brite brown adipocytes - a newly identified type of brown adipocyte distinct from the classical brown adipocyte that makes up the interscapular thermogenic organ of other mammals. A combination of high resolution imaging techniques, histological and biochemical analyses enabled us to provide evidence for an anatomically distinguishable interscapular BAT depot in human infants that consists of classical brown adipocytes, a cell type so far not proven to exist in humans. Based on these findings, we conclude that infants, as rodents, possess the bona fide interscapular BAT thermogenic organ made up by classical brown adipocytes essential for the survival of small mammals in a cold environment.

Based on the seminal observation by Cannon and Nedergaard ${ }^{1}$ that human PET scans sometimes depicted a symmetric cold induced uptake of FDG-glucose, three independent studies, published in April 2009, demonstrated metabolically highly active brown adipose tissue (BAT) in adult humans ${ }^{2-4}$. Subsequent investigations demonstrated an inverse association of obesity and type 2 diabetes mellitus and the presence of active $\mathrm{BAT}^{5-7}$. A unique characteristic of BAT is the expression of uncoupling protein 1 (UCP1, also known as thermogenin). Activation of this transmembrane protein by fatty acids in response to adrenergic signaling short-circuits the inner mitochondrial membrane's proton gradient thereby uncoupling oxidative phosphorylation from ATP synthesis. Hence, chemical energy stored in the gradient is dissipated as heat allowing for efficient direct thermogenesis without shivering ${ }^{8}$. This adaptive defense against cold has been examined extensively in rodents and many aspects of BAT development and function have been elucidated. In rodents it is evident 
that not only the distinct thermogenic BAT organ located in the interscapular region (iBAT) consists of brown adipocytes, but that a second type of brown adipocytes, so-called beige or brite cells can appear in white adipose tissue (WAT) depots in response to cold or $\beta 3$ adrenergic stimuli ${ }^{9,10}$. Recently, lineage tracing experiments revealed that the two cell types have a different developmental origin ${ }^{11}$. While classical brown adipocytes and skeletal muscle cells arise from precursors in the dermomyotome ${ }^{12}$, beige/brite cells seem to originate from endothelial and perivascular cells within WAT depots ${ }^{13-15}$. A recent study by Wu et al suggests that the previously described depots of human BAT are of the beige/brite type and raises the question whether humans altogether lack classical brown adipocytes ${ }^{16}$, this has also been the topic of a recent review ${ }^{17}$. Histomorphological studies performed in the 1970s indicated the existence of brown adipocytes within the interscapular region in human infants and that these disappeared with age ${ }^{18}$. Using a combination of high resolution imaging techniques and morphological and biochemical analyses, we tested the hypothesis that human infants, like small mammals, possess an anatomically distinguishable iBAT depot consisting of classical brown adipocytes, a cell type so far not proven to exist in humans.

In an attempt to visualize potential iBAT in humans we performed post mortem MR imaging of eight human infants. Using the fat fraction method ${ }^{19}$ we did not only identify BAT depots in the supraclavicular region, but importantly also a fat depot in the interscapular region presenting with an intermediate fat fraction as opposed to the high fat fraction of the surrounding subcutaneous WAT (Supplementary Fig. 1). Using a three dimensional reconstruction we were able to compute the volume of the tissue depot with an average $( \pm \mathrm{SD})$ volume of 3.6 $\pm 2.4 \mathrm{ml}$. Figure 1 displays a representative reconstruction of the iBAT depot. As visualized in the MRI reconstruction, the tissue exhibits an island-like distribution in the 
interscapular region. However, the existence of thin connections between the islands of tissue below the resolution limit of the imaging technique used cannot be excluded.

In order to unequivocally confirm the BAT identity of the imaged tissue in the interscapular region, image guided biopsies were taken from four subjects and analyzed following hematoxylin-eosin and immunofluorescence staining. The sampled tissue consisted of packed multilocular brown adipocytes with very few interspersed unilocular UCP1-negative adipocytes and was delineated from the subcutaneous WAT by a layer of connective tissue (Supplementary Fig. 2a). The immunofluorescent staining for UCP1 and the mitochondrial marker, cytochrome c oxidase revealed a high density of mitochondria with a near perfect colocalization of UCP1, thus confirming that the sampled tissue indeed was BAT (Supplementary Fig. 2b). In order to compare the appearance of the interscapular BAT to that of other depots, we acquired BAT samples from two other locations and studied their histological features. Like the interscapular BAT, samples from the periadrenal region of patients undergoing surgery for benign adrenal tumors and from the supraclavicular region of healthy volunteers displayed mitochondria rich and strongly UCP1 positive multilocular adipocytes (Supplementary Fig. 2b). However, in these two groups, the brown adipocytes were interspersed as clusters between unilocular UCP1 negative adipocytes (Supplementary Fig. 2a,b).

Based on the available literature on BAT marker genes we identified Zicl as being the gene that best discriminates $\mathrm{BAT}$ from beige/brite $\mathrm{BAT}$ in mice. ZIC1 is a transcription factor involved in neurogenesis and myogenesis and its overexpression is associated with liposarcomas in humans ${ }^{20}$. Its expression is virtually absent in murine beige/brite adipose tissue but readily present in iBAT as detected by quantitative real-time $\mathrm{PCR}^{21}$. We therefore 
performed immunofluorescence for ZIC1 in sections of human BAT. While the interscapular BAT displayed a distinct ZIC1 staining, the brown adipocytes of the supraclavicular and periadrenal BAT depots only showed a faint expression of ZIC1 (Supplementary Fig. 2c). In line with the immunofluorescence results we could show that the ZIC1 mRNA expression level indeed was highest in human BAT sampled from the interscapular region and was approximately 100-fold higher than in supraclavicular BAT $(\mathrm{p}<0.01)$ and 10 -fold higher than in periadrenal BAT $(\mathrm{p}<0.05)($ Fig. 2a). From one of the infant subjects an image guided biopsy was taken also from the perirenal region. This sample was grouped together with the adult periadrenal samples and the expression level of ZIC1 in this tissue was well in range with the level in adult periadrenal samples. For this specific subject the expression level of ZIC1 was approximately 6-fold higher in interscapular BAT compared to BAT from the perirenal region (red data points in Fig. 2a). This observation is important since it indicates that in infants, the level of ZIC1 expression is higher in interscapular classical BAT as compared with other depots, a situation very similar to that in mice ${ }^{21}$. This observation is then further extended when a significant depot specific difference is observed with regard to ZICI expression when adult human BAT depots are compared with infant interscapular BAT (Fig. 2a).

To further characterize and compare the BAT samples from the different depots we performed a more extensive gene expression analysis. Using clonal cell lines, Wu et al described further potential marker genes for classical and beige/brite BAT in mice ${ }^{16}$. Among those, Epithelial V-like Antigen 1 (Eval) was suggested as a good marker for classical BAT, while Tbxl and Tmem 26 appeared to be reliable markers of murine beige/brite BAT. In the human samples analyzed here, EVA1 displayed a significantly higher expression level in periadrenal BAT as compared to iBAT $(\mathrm{p}<0.05)$ and supraclavicular BAT $(\mathrm{p}<0.01)$ (Fig. 2b). The expression of 
$T B X 1$ was about 10 -fold higher in the supraclavicular as compared to the iBAT samples $(\mathrm{p}<0.05)$ (Fig. 2c). The TBX1 expression in iBAT and periadrenal BAT did not differ significantly. For TMEM26 no significant differences between the three groups could be detected (Fig. 2d). Thus, in humans both ZIC1 and TBX1 appear to be markers that in a significant way discriminate between BBAT and BAT from other depots, which is in accordance with results derived from studies in mouse ${ }^{16,21}$.

In mice, the homeobox genes $H o x c 9$ and Shox 2 are differentially expressed in retroperitoneal beige/brite BAT as compared to BBAT or other beige/brite or white depots. Hoxc 9 expression is virtually absent in murine iBAT and two-fold higher in the retroperitoneal than in the inguinal subcutaneous depot. Shox 2 expression on the other hand is undetectable in murine retroperitoneal beige/brite fat and iBAT but readily detectable in the inguinal subcutaneous $\operatorname{depot}^{21}$. In the human samples presented here HOXC9 expression was approximately 5 -fold higher in the periadrenal BAT $(\mathrm{p}<0.001$ versus supraclavicular and $\mathrm{p}<0.05$ versus iBAT) (Fig. 2e) and $\mathrm{SHOX} 2$ was approximately 5-fold lower in periadrenal as compared to supraclavicular BAT $(\mathrm{p}<0.01)$ (Fig. 2f), thus reflecting the findings in mice.

The expression of UCP1 (Fig. 2g), the hallmark of BAT, was comparable between the three groups as were the expression levels of two other genes characteristic of BAT, deiodinase type II (DIO2) and $\beta 3$-adrenergic receptor $(A D R B 3)$ (Fig. 2h,i). While the global Kruskal Wallis test indicated significant differences in the expression level of $A D R B 3$ between the three groups, the pairwise comparisons by Mann Whitney U tests followed by Bonferroni Holm correction did not reach statistical significance. The two transcription factors, PR domain containing 16 (PRDM16) and peroxisome-proliferator-activated receptor $\gamma$ coactivator $1 \alpha(P P A R G C 1 A)$, playing crucial roles during the differentiation of brown adipocytes were also expressed to a similar degree in all three BAT groups (Fig. $\mathbf{2 j} \mathbf{j}, \mathbf{k})$. 
Guided by MR imaging we have been able to acquire BAT samples from the interscapular region of 4 human infants, an anatomical localization identical to that occupied by classical BAT in previously studied mammals such as mice and rats. The histomorphology of the human interscapular BAT closely resembles the typical iBAT depot found in rodents. Densely packed brown adipocytes and only few interspersed white adipocytes are separated from the surrounding WAT by a layer of connective tissue. In contrast, the samples taken from the periadrenal and supraclavicular regions of human adults show a pattern of prevailing unilocular white adipocytes with interspersed clusters of multilocular UCP1 expressing brown adipocytes similar to the clusters of beige/brite BAT appearing in WAT depots of cold exposed rodents ${ }^{10}$. Previous morphologic studies indicate a regression of the human iBAT depot during infancy ${ }^{18}$, which is in line with the results of FDG-PET/CT studies in human adults that display avid tracer uptake in the supraclavicular region but not in the interscapular $\operatorname{area}^{22}$. The measured volume of the human infant iBAT depot in relation to total body volume is comparable in order of magnitude to the volume of supraclavicular BAT depot determined in adults ${ }^{4}$.

These morphologic findings are corroborated by the gene expression analysis of infant iBAT. The transcription factor Zic1 is a highly specific marker for classical BAT depots, which are of myogenic lineage in mice ${ }^{21}$. We show that the expression level of this iBAT marker is one to two orders of magnitude higher in human interscapular BAT than in human supraclavicular and periadrenal BAT. The high expression of ZICl in the human BBAT depot strongly suggests a myogenic origin of this tissue, analogous to the situation in rodents. In contrast, the presumed iBAT marker EVAl was not preferentially expressed in the human iBAT as compared to the beige/brite BAT depots. However, in mice Zicl had a much greater 
discriminatory power being more than 1000-fold different between iBAT and beige/brite BAT, while Eval was only about 5-fold different. In agreement with data presented by Wu et al, the beige/brite marker $T B X 1$ was expressed at a significantly higher level in supraclavicular than in interscapular BAT, underscoring the different characteristics of these two BAT depots. No difference in TBXI expression could be detected between the iBAT and periadrenal BAT. This is in line with our own data from mice (data not shown), which indicate that TBX1 may be a preferential marker for subcutaneous beige/brite depots rather than all beige/brite depots. We also show that the retroperitoneally located periadrenal depot expressed high levels of $\mathrm{HOXC} 9$ and low levels of $\mathrm{SHOX} 2$ which reflects the findings in murine retroperitoneal versus inguinal/subcutaneous beige/brite adipose tissue ${ }^{21}$. Importantly, the expression level of $U C P 1$ as well as that of other BAT associated genes (DIO2, PRDM16 and PPARGC1A) was comparable in the examined human BAT depots suggesting a functional equivalence of the tissues.

Ideally, the comparison between different depots of human infant BAT should be performed with samples that are derived from matched (gender, age, body weight etc.) subjects. In reality however this is extremely difficult - for obvious reasons biopsies of the kind used in this report are exceedingly hard to obtain. Although there are some differences, as one would expect, the gene expression data presented here that is derived from genetically divergent humans are remarkably similar to what has been observed regarding differences between classical iBAT and beige/brite BAT using genetically constant inbred mice ${ }^{16,21}$. With this in mind and in support of the conclusions made here we would like to high-light the following: First, as in mice, ZICl expression is significantly higher in human classical interscapular BAT as compared with other depots ${ }^{21}$. Second, the $T B X 1$ expression is significantly higher in supraclavicular BAT as compared with BBAT which is in line with a previous study 
performed on mice ${ }^{16}$. Third, the depot specific expression of HOXC9 and SHOX2 agrees well with previous studies in mice ${ }^{21}$. Fourth, we demonstrate an anatomical localization of human classical iBAT identical to that of previously studied mammals such as mice and rats. While previous studies have demonstrated an interscapular tissue with many of the characteristic features of $\mathrm{BAT}^{18,23}$, we can, by using advanced imaging and molecular analyses, provide evidence for the presence of bona fide interscapular BAT in human infants that consists of classical BAT cells as opposed to beige/brite brown adipocytes. Recently, the depiction of supraclavicular BAT by MRI in human infants has been described in a single case ${ }^{19}$. To our knowledge, we provide the first report of reliable MRI based detection of iBAT in humans.

The fact that different populations of brown adipocytes exist in humans is remarkable as these might be differently affected by external stimuli and represent distinct potential targets for pharmacological intervention. Thus, reactivation of BAT in adult humans constitutes an alternative approach to that of stimulation of beige/brite brown adipocytes.

\section{ACKNOWLEDGEMENTS}

This work was supported by grants to SE from the Swedish Research Council (grant numbers 2009-2590 and 2010-3281), The Knut and Alice Wallenberg Foundation, Sahlgrenska's University Hospital (LUA-ALF), EU grants (LSHM-CT-2003-503041; DIABESITY and HEALTH-F2-2011-278373; DIABAT), The IngaBritt and Arne Lundgren Foundation, The Söderberg Foundation, and the Swedish Foundation for Strategic Research through the Center for Cardiovascular and Metabolic Research. MJB was supported by a postdoctoral fellowship from the Fritz Thyssen-Stiftung. We would like to thank Brigitte Mauracher (Medizinische Klinik und Poliklinik IV, Klinikum der LMU, Munich) for excellent technical assistance and 
acknowledge the Centre for Cellular Imaging and the Genomics Core Facility at Sahlgrenska Academy for technical assistance and use of equipment.

\section{AUTHOR CONTRIBUTIONS}

A.P., M.B., M.E.L., M.J.B. and S.E. conceived and designed the experiments. D.N., L.E., M.E.L., M.H., M.J.B., O.D.L. and T.R. performed the experiments. A.P., D.N., L.E., M.B., M.E.L., M.H., M.J.B., O.D.L., S.E. and T.R. analyzed the data. F.B., K.A.V., M.S., T.M. and P.N. provided samples. M.E.L., M.J.B. and S.E. wrote the manuscript.

\section{FIGURE LEGENDS}

Figure 1 Three-dimensional reconstruction of interscapular BAT of a human infant. Representative three-dimensional reconstruction of the interscapular BAT of a human infant. The volume rendered data set is shown in the sagittal (a), coronal (b), and axial plane (c). The interscapular BAT is colored in green.

Figure 2 Gene expression profiling of human brown adipose tissue depots. Quantitative realtime PCR was performed on supraclavicular, interscapular and periadrenal brown adipose tissue using primers detecting genes suggested to be either preferentially expressed in classical brown adipocytes (a,b; ZICl and EVA1) or beige/brite cells (c-f; TBX1, HOXC9, SHOX2 and TMEM26) or in brown adipose tissue in general (g-k; UCP1, DIO2, ADRB3, PRDM16 and PPARGC1A). Expression levels were normalized to that of $A C T B$. The red data points in $\boldsymbol{a}$ corresponds to the ZIC1 levels measured in interscapular and perirenal samples from the infant subject from which both types of biopsies were taken. The global P value of the Kruskal-Wallis test is given above the respective graph. ${ }^{*} \mathrm{P} \leq 0.05, * * \mathrm{P} \leq 0.01$ and ***P $\leq 0.001$ for pairwise comparison by Mann-Whitney $\mathrm{U}$ test. 


\section{METHODS}

\section{Subjects and biopsy procedures}

Eight human infants, age between 0 and 74 weeks, underwent MRI 24 to 48 hours post mortem for identification of BAT. The core body temperatures were approximately $+5^{\circ} \mathrm{C}$ during scanning. After image reconstruction, BAT-like tissue was identified on the basis of an intermediate fat-signal fraction in between that of muscle and white adipose tissue as has been discussed previously ${ }^{19}$. From four of the subjects (age between 3 and 14 days), biopsies could be taken from the interscapular BAT depot identified by the MRI and from one of the subjects an MRI guided biopsy could also be taken from the perirenal region. The biopsies were stored in RNAlater (Qiagen) at $+4^{\circ} \mathrm{C}$ until further processed. The MRI and acquisition of biopsies were approved by the Regional Ethical Committee in Linköping (RMV M64-05 and 75-05). The subjects and procedure for biopsy acquisition of supraclavicular BAT from healthy adults have been previously described ${ }^{24}$.

Periadrenal BAT samples from fifteen patients undertaking surgery for benign adrenal tumors were evaluated for this study. The tumor and adjacent adipose tissue was removed using an endoscopic retroperitoneal approach, according to standard procedures and immediately placed on ice. After sampling and assessment of the specimen by a pathologist the remaining tumor and adjacent fat was partitioned and frozen in liquid nitrogen. The adipose tissue adjacent to the tumor was used for this study. The procedure was approved by the Institutional Review Board of the Ludwig-Maximilian-University, Munich (project number 379-10), and all patients provided written informed consent.

\section{Magnetic resonance imaging}

Post-mortal MRI was acquired using a 3.0 T Ingenia clinical MR-scanner (Philips). A phased array head/neck coil was used for acquisition of symmetrically sampled 3D gradient dual echo 
2-Point Dixon images ${ }^{25}$ with echo times of approximately $3.30 \mathrm{~ms}$ (opposite phase image) and $6.60 \mathrm{~ms}$ (in-phase image). The repetition time was $9.3 \mathrm{~ms}$ and the flip angle was 10 degrees. The obtained resolution was $0.75 \times 0.75 \times 0.75 \mathrm{~mm}^{3}$. Fat and water separated images were obtained using the inverse gradient method ${ }^{26,27}$ and image inhomogeneity was corrected for by normalization of an interpolated bias field from identified pure adipose tissue voxels (for correction of the fat image) and pure muscle tissue voxels (for correction of the water image $)^{28,29}$.

\section{Three-dimensional reconstruction of MR images}

The interscapular BAT was segmented in the MR images, using a segmentation tool based on the Image Foresting Transform ${ }^{30}$. The identification of BAT was based on the fat-water fraction in the images. In a representative subject the scapulae and vertebrae were manually segmented in order to facilitate the visualization. This data set was volume rendered in three different orientations with the interscapular BAT colored green. The volumes of the segmented interscapular BAT depots were calculated in Matlab software (MathWorks Inc) by calculating the number of voxels in each segmented region and multiplying with the voxel size.

\section{Morphological and immunohistochemical studies}

Frozen samples were cut in $10 \mu \mathrm{m}$ sections on a CM3050 cryostat (Leica) and mounted on positively charged glass slides (Thermo Fisher Scientific). Sections were fixed in ice-cold acetone for 7 min and subsequently brought to water by ethanol dilution series. Hematoxylin and Eosin (HE) staining was performed according to standard procedures. 
Immunofluorescence for UCP1 was performed as follows: slides were incubated in blocking buffer consisting of Dulbecco's Modified Eagle Medium (Life Technologies) supplemented with $10 \%$ fetal bovine serum (Life Technologies) for 1 hour at room temperature. A rabbit polyclonal antibody directed against UCP1 (Sigma, U6382) was used at a dilution of 1:500 in blocking buffer and slides were incubated with the primary antibody at $+4^{\circ} \mathrm{C}$ overnight. Slides were subsequently washed with phosphate buffered saline (PBS) and incubated with Alexa Fluor 488 conjugated anti-rabbit IgG secondary antibody (1:500 dilution, Life Technologies, Molecular Probes), To-Pro3 nuclear stain (Molecular Probes, 1:1000), and Alexa594 conjugated mouse anti-OxPhos Complex IV subunit I (1:200, Life Technologies, Molecular Probes A21297) for two hours at room temperature. After final washes in PBS, slides were mounted using ProLong Gold Antifade Reagent (Life Technologies, Molecular Probes). For ZIC1 immunofluorescence a rabbit polyclonal antibody (Abcam ab72694) was used at a dilution of 1:50 at $4{ }^{\circ} \mathrm{C}$ overnight. Detection was performed with an AlexaFluor568 conjugated anti-rabbit IgG antibody. For co-staining against UCP1 the above mentioned antibody was directly labeled with a Zenon rabbit IgG AlexaFluor 488 labeling kit (Life Technologies, Molecular Probes) and used at a dilution of 1:250 for $2 \mathrm{~h}$ at RT.

HE stained slides were imaged on an Eclipse E800 microscope (Nikon). Immunofluorescence was visualized on an LSM 510 Meta system (Zeiss).

\section{Laser microdissection}

For three of the post-mortem biopsies of interscapular BAT a laser microdissection (LMD) approach was used to avoid contamination of the sample by adjacent connective tissue and muscle; the fourth sample was of such character that BAT could be microdissected without contamination of adjacent tissues using a binocular microscope. For LMD a PALM microbeam workstation (Zeiss) was used. Briefly, $10 \mu \mathrm{m}$ cryo-sections were mounted on 
PALM PEN MembraneSlides (Zeiss) and subjected to cresyl violet staining (Sigma) according to the manufacturer's recommendations. A final dehydration and de-fatting step using $100 \%$ acetone was added to the protocol. Adjacent sections were mounted on positively charged glass slides and immunofluorescence for UCP1 was performed to confidently identify BAT.

\section{Biochemical studies}

Total RNA was isolated from the biopsies using the RNeasy Lipid Tissue Mini Kit (Qiagen) or in the case of the LMD samples, with the RNeasy plus micro kit (Qiagen). cDNA synthesis was performed using the 1st Strand cDNA Synthesis Kit for RT-PCR (AMV) (Roche). Quantitative real-time PCR was performed on an ABI PRISM 7900HT instrument (Applied Biosystems) using Power SYBR Green PCR Master Mix (Applied Biosystems). The standard curve method with $A C T B$ as the normalizing gene was used to determine relative gene expression levels. Primers were designed to span intron/exon borders (sequences are supplied in Supplementary Table 1).

\section{Statistical analysis}

As not all gene expression data could be assumed to follow a normal distribution, statistical analysis was performed with GraphPad Prism Version 5.04 (GraphPad) using the KruskalWallis test followed by Mann-Whitney U-tests. The family-wise type I error was accounted for by Bonferroni-Holm correction. 


\section{REFERENCES}

1. Nedergaard, J., Bengtsson, T. \& Cannon, B. Am. J. Physiol. Endocrinol. Metab. 293, E444-452 (2007).

2. Cypess, A.M. et al. N. Engl. J. Med. 360, 1509-1517 (2009).

3. van Marken Lichtenbelt, W.D. et al. N. Engl. J. Med. 360, 1500-1508 (2009).

4. Virtanen, K.A. et al. N. Engl. J. Med. 360, 1518-1525 (2009).

5. Lee, P., Greenfield, J.R., Ho, K.K. \& Fulham, M.J. Am. J. Physiol. Endocrinol. Metab. 299, E601-606 (2010).

6. $\quad$ Ouellet, V. et al. J. Clin. Endocrinol. Metab. 96, 192-199 (2011).

7. Pfannenberg, C. et al. Diabetes 59, 1789-1793 (2010).

8. Berg, J.M., Tymoczko, J.L. \& Stryer, L. Biochemistry, (W. H. Freeman, 2007).

9. Cousin, B. et al. J. Cell Sci. 103, 931-942 (1992).

10. Guerra, C., Koza, R.A., Yamashita, H., Walsh, K. \& Kozak, L.P. J. Clin. Invest. 102, 412-420 (1998).

11. Seale, P. et al. Nature 454, 961-967 (2008).

12. Atit, R. et al. Dev. Biol. 296, 164-176 (2006).

13. Gupta, R.K. et al. Cell Metab. 15, 230-239 (2012).

14. Lee, Y.H., Petkova, A.P., Mottillo, E.P. \& Granneman, J.G. Cell Metab. 15, 480-491 (2012).

15. Tran, K.V. et al. Cell Metab. 15, 222-229 (2012).

16. Wu, J. et al. Cell 150, 366-376 (2012).

17. Cannon, B. \& Nedergaard, J. Nature 488, 286-287 (2012).

18. Heaton, J.M. J. Anat. 112, 35-39 (1972).

19. Hu, H.H., Tovar, J.P., Pavlova, Z., Smith, M.L. \& Gilsanz, V. J. Magn. Reson. Imaging 35, 938-942 (2012). 
20. Brill, E. et al. Cancer Res. 70, 6891-6901 (2010).

21. Walden, T.B., Hansen, I.R., Timmons, J.A., Cannon, B. \& Nedergaard, J. Am. J. Physiol. Endocrinol. Metab. 302, E19-31 (2012).

22. Enerback, S., Oksi, J. \& Nuutila, P. N. Engl. J. Med. 361, 420-421 (2009).

23. Houstek, J. et al. J. Clin. Endocrinol. Metab. 77, 382-387 (1993).

24. Orava, J. et al. Cell Metab. 14, 272-279 (2011).

25. Dixon, W.T. Radiology 153, 189-194 (1984).

26. Rydell, J. et al. Medical image computing and computer-assisted intervention : MICCAI ... International Conference on Medical Image Computing and ComputerAssisted Intervention 10, 210-218 (2007).

27. Romu, T., Dahlqvist Leinhard, O., Lundberg, P. \& Borga, M. Robust fat-water separation of symmetrically sampled two point Dixon data. in ISMRM workshop on Fat-Water Separation: Insights, Applications \& Progress in MRI (Long Beach, CA, USA, 2012).

28. Leinhard, O.D. et al. Quantitative abdominal fat estimation using MRI. in International Conference on Pattern Recognition art. no. 4761764 (2008).

29. Romu, T., Borga, M. \& Dahlqvist, O. MANA - Multi scale adaptive normalized averaging. in International Symposium on Biomedical Imaging art. no. 5872424 (2011).

30. Malmberg, F., Lindblad, J. \& Nyström, I. Sub-pixel Segmentation with the Image Foresting Transform. in 13th International Workshop on Combinatorial Image Analysis (IWCIA'09) (Playa del Carmen, Mexico, 2009). 


\section{a}

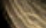

कh

y $x^{2}-3$

1. $x-23$

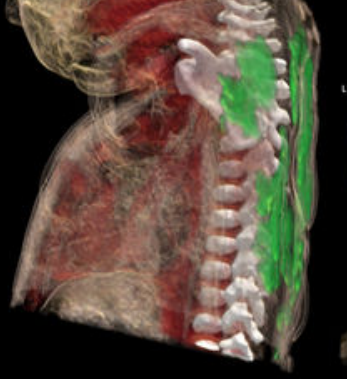

b 12

$\mathrm{t}$

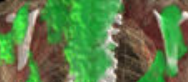

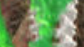

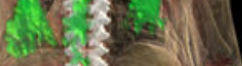

.

c 
a

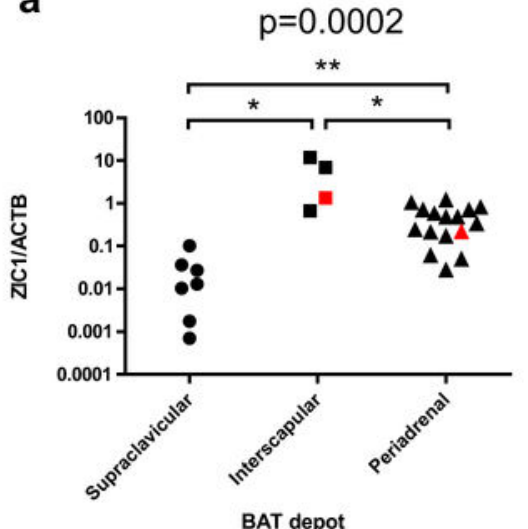

d

NS

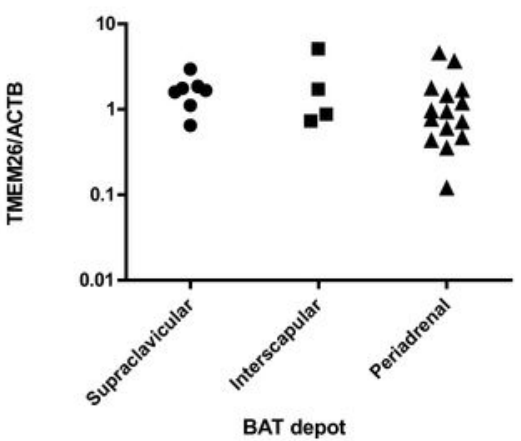

g

NS

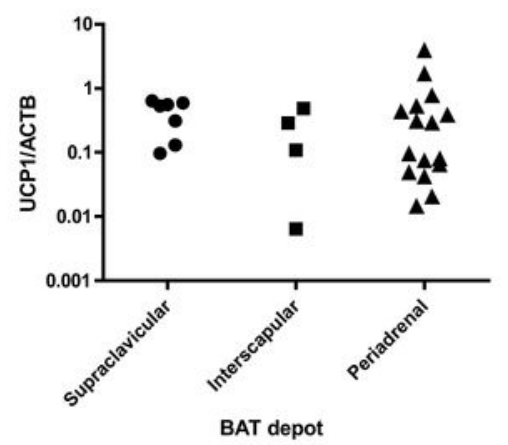

j

NS

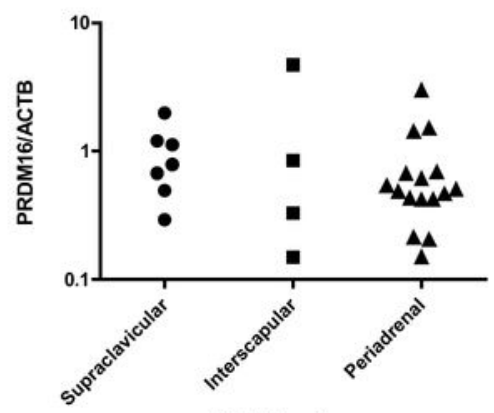

BAT depot

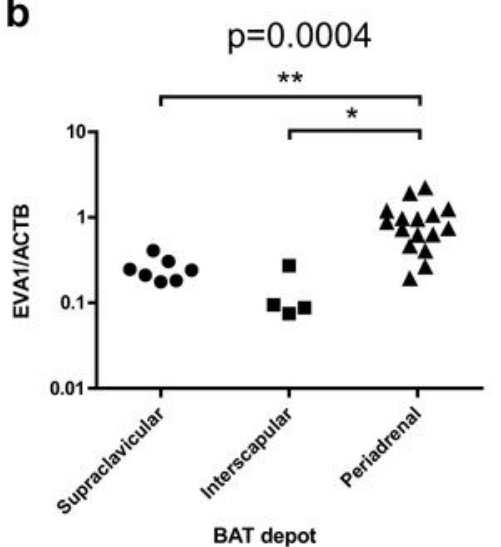

e

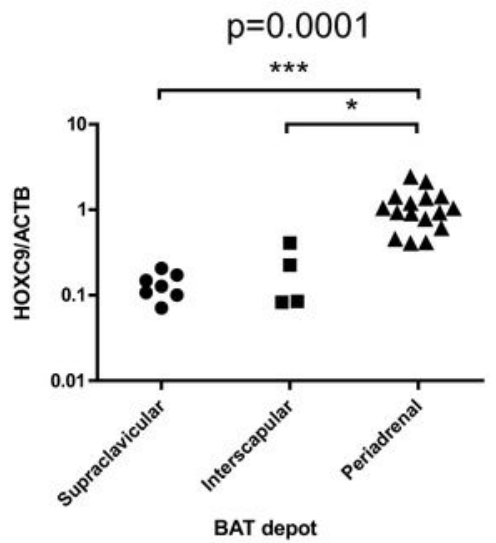

h

NS

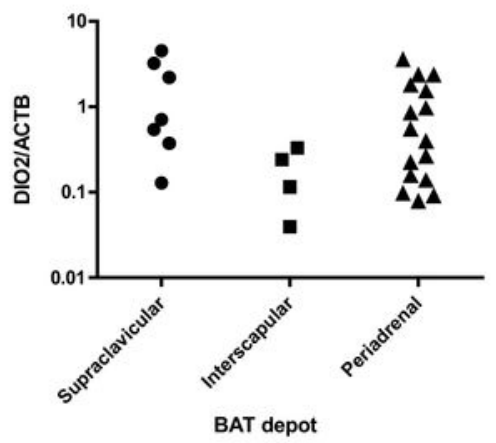

k

NS

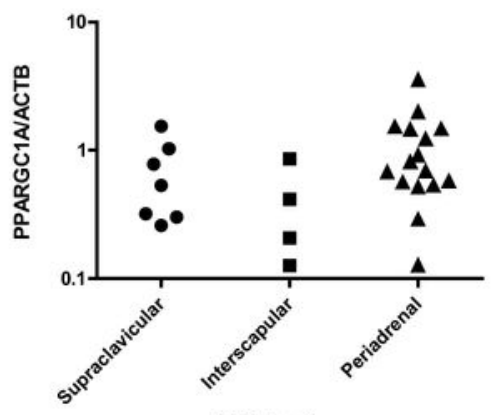

C $p=0.0038$

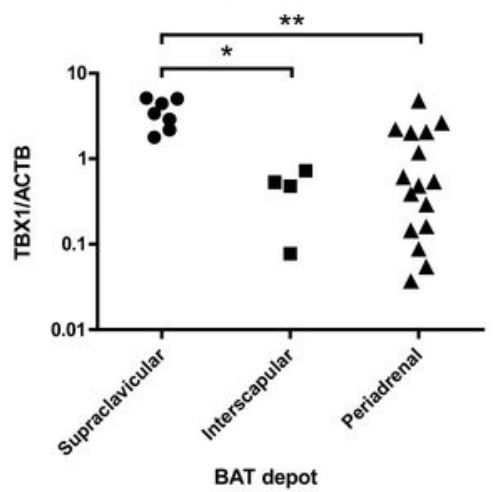

f

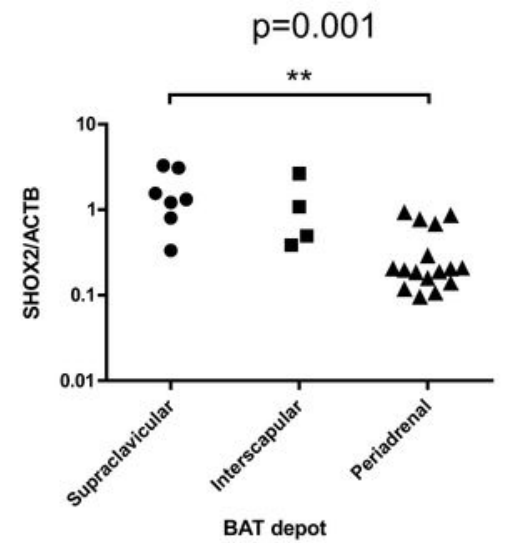

i

$p=0.0258$

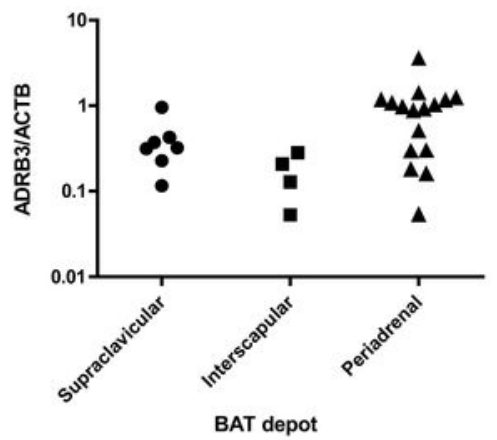
BAT depot 


\section{SUPPLEMENTARY INFORMATION}

Lidell et al.

Evidence for Two Types of Brown Adipose Tissue in Humans

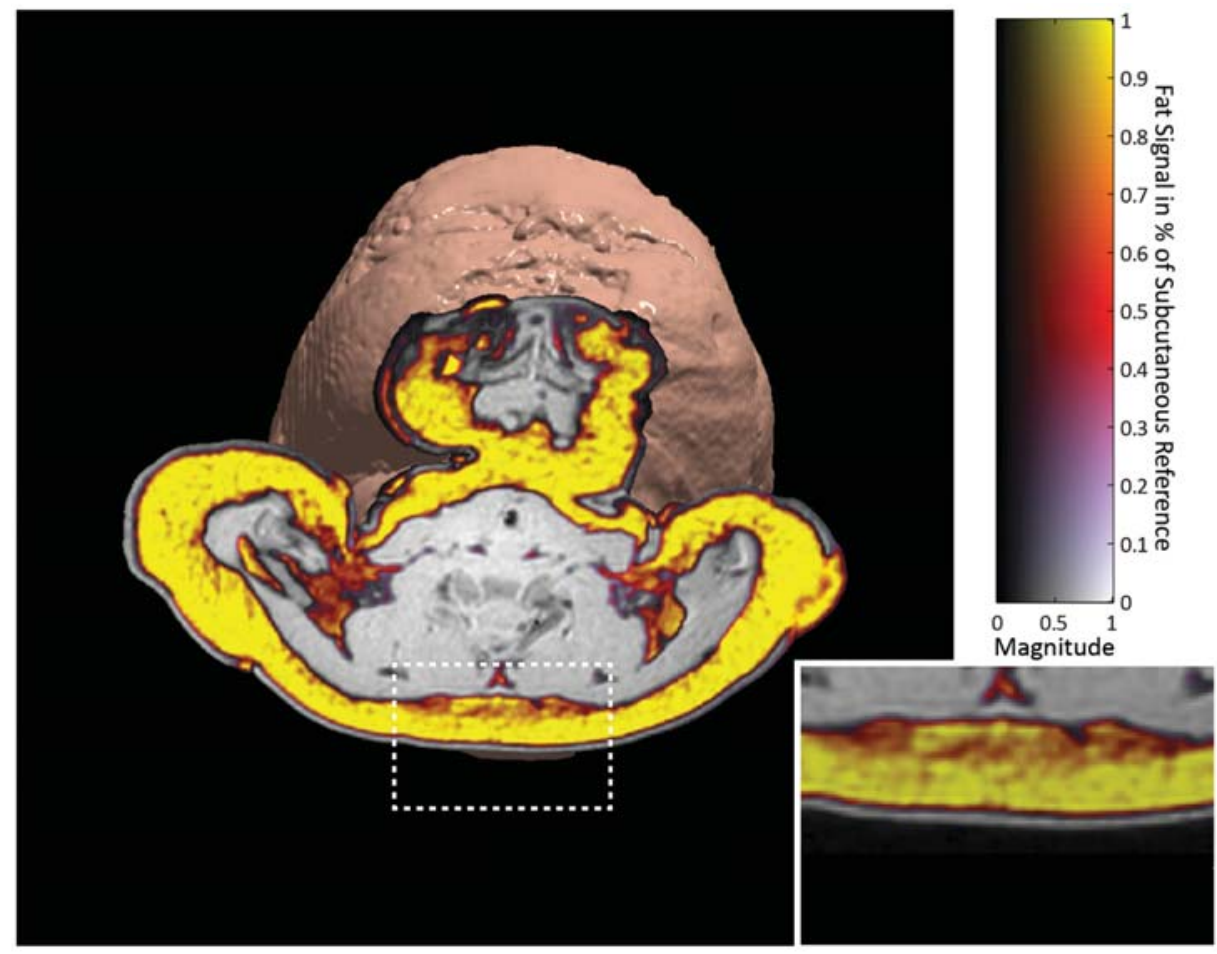

Supplementary Figure 1 Human infants possess an anatomically distinguishable interscapular brown adipose tissue depot. Post mortem MRI of a human infant. In the clip plane, a gray scale anatomical image is shown with a color overlay created using a linear mapping of the lipid signal from 0 , corresponding to lipid signal strength in muscle tissue, to 1 , corresponding to lipid signal strength in identified pure adipose tissue. The region containing interscapular brown adipose tissue is highlighted and magnified to the right in the figure. 


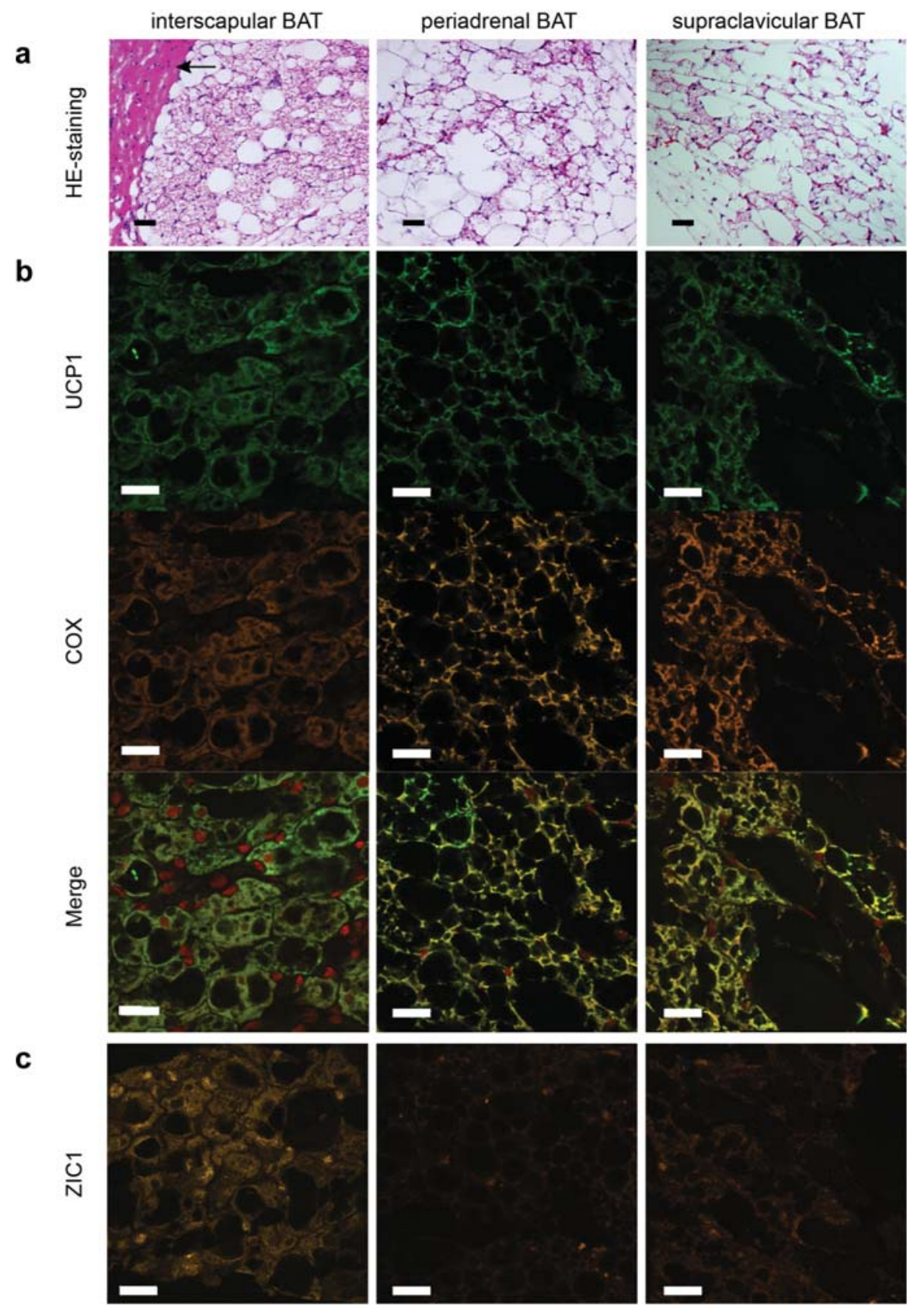

Supplementary Figure 2 Brown adipose tissue depots identified by MRI represent bona fide brown adipose tissue built by classical brown adipocytes. Sections of interscapular, periadrenal and supraclavicular brown adipose tissue were hematoxylin-eosin (HE) stained (a) or used for immunofluorescence staining (b-c). The sections were either co-stained using antibodies against UCP1 (green) (b; upper panel) and the mitochondrial marker cytochrome $\mathrm{c}$ oxidase (COX; orange) (b; middle panel) or with an antibody against ZIC1 (orange) (c). A merged image (Merged) of the co-stained sections is shown (b; lower panel). Nuclei were stained with To-Pro3 (red). Black scale bars are $50 \mu \mathrm{m}$ and white scale bars are $20 \mu \mathrm{m}$. The arrow marks the connective tissue delineating the interscapular brown adipose tissue from the adjacent white adipose tissue. 
Supplementary Table 1. Sequences of primers used for quantitative real-time PCR

\begin{tabular}{|l|l|l|}
\hline Gene & Forward Primer Sequence & Reverse Primer Sequence \\
\hline ADRB3 & TTTGCCAACGGCTCGAC & CGTCAGGTTCTGGAGGGTAG \\
\hline ACTB & GAGCTACGAGCTGCCTGACG & GTAGTTTCGTGGATGCCACAG \\
\hline DIO2 & CCTCCTCGATGCCTACAAAC & GCTGGCAAAGTCAAGAAGGT \\
\hline EVA1 & TAGGGGAGATCCGGCTCAGCG & GCCAATGGCCAGAGCCAGGAAG \\
\hline HOXC9 & GCAGCAAGCACAAAGAGGAGA & GCGTCTGGTACTTGGTGTAGGG \\
\hline PPARGC & GCCAAACCAACAACTTTATCTCT & CACACTTAAGGTGCGTTCAATAG \\
\hline PRDM16 & GAGGAGGACGATGAGGACAG & CGGCTCCAAAGCTAACAGAC \\
\hline SHOX2 & GGCCCGAGTGCAGGTTTGGT & AACTGGCTGGCGGCCCCTAT \\
\hline TBX1 & AGTTCAACCAGCTGGGCACCG & GGCCATGGGATCCATGCCGAA \\
\hline TMEM26 & GTTCAAGCGCGGACGAGGCT & AAGCCACAGGGACGGCATGA \\
\hline CTGGAATAGCGGCGTGCTT & AATAACACTGGACGTCGGGC \\
\hline
\end{tabular}

